\title{
Hyperbolicity of the BSSN system of Einstein evolution equations
}

\author{
Olivier Sarbach, Gioel Calabrese, Jorge Pullin, and Manuel Tiglio \\ Department of Physics and Astronomy, Louisiana State University, \\ 202 Nicholson Hall, Baton Rouge, Louisiana 70803-4001
}

\begin{abstract}
We discuss an equivalence between the Baumgarte-Shapiro-Shibata-Nakamura (BSSN) formulation of the Einstein evolution equations, a subfamily of the Kidder-Scheel-Teukolsky formulation, and other strongly or symmetric hyperbolic first order systems with fixed shift and densitized lapse. This allows us to show under which conditions the BSSN system is, in a sense to be discussed, hyperbolic. This desirable property may account in part for the empirically observed better behavior of the BSSN formulation in numerical evolutions involving black holes.
\end{abstract}

\section{INTRODUCTION}

When numerically integrating the Einstein equations, it is common to split them into constraint and evolution equations. Most attempts usually solve the "free evolution" problem, solving the constraints only at the level of initial data. As in all gauge theories, the evolution equations of general relativity are not uniquely defined. One can redefine them by adding constraints, since the latter vanish on solutions of the whole system of equations. It has been increasingly recognized in the last few years (see [1] for a list of references), that it is desirable to take advantage of this freedom to make the evolution equations strongly- (and preferably symmetric-) hyperbolic. There is a body of mathematical literature [2] that one can draw from to prove stability (in the sense of Lax's theorem) for discretization schemes based on well posed formulations. In hyperbolic formulations one also has better knowledge of the modes of the equations, something that is of interest at the time of specifying boundary conditions that are consistent with the constraints and the evolution equations [3, 4].

The most traditional of the evolution schemes, the Arnowitt-Deser-Misner (ADM) scheme, when written in first order form, is only weakly hyperbolic (WH) [5]. There has always been a feeling that this accounted in part for the difficulties encountered trying to evolve black holes and other spacetimes with this evolution scheme. More recently [6], it was rigorously shown that popular discretizations of the ADM equations in particular, and WH formulations in general, simply do not lead to convergent schemes.

It was first recognized by Shibata and Nakamura [7] and further elaborated by Baumgarte and Shapiro [8] that if one performed a conformal-traceless (CT) decomposition of the ADM variables, the resulting scheme (commonly known as BSSN), at least in certain situations, appeared to have less numerical difficulties than the ADM system. Also, anecdotal evidence suggested that slightly different implementations of the BSSN scheme seemed to perform quite differently in numerical simulations.

In this paper we would like to point out that the BSSN scheme, under certain conditions, is a reduction (from first to second order in space) of some strongly or symmetric hyperbolic systems. This may account in part for its better performance in numerical simulations. The conditions under which these reductions can be established depends rather subtly on details of the implementation of the BSSN scheme, this may account for why some implementations appear better behaved than others.

We will show that the BSSN scheme and its generalization by Alcubierre et. al. [9] can be mapped to the KidderScheel-Teukolsky (KST) [5] system of evolution equations in the linearized regime. The latter is a multi-parameter family of evolution schemes, and we will discuss for which values of the parameters the correspondence holds. The BSSN system is a first order in time and second order in space formulation, whereas the KST system is first order in time and space. Therefore we will need to perform an enlargement of the BSSN system to establish the correspondence. We will discuss in detail the precise nature of the correspondence below. We will show that a similar enlargement exists at the nonlinear level, which does not yield the KST system but which does yield a strongly or symmetric hyperbolic one. The latter system is closely related to one introduced by Friedrich and Rendall [10].

We will start by presenting, in Section II, the KST system and summarizing their main properties. Next we present a CT decomposition of this system that shares its level of hyperbolicity. We then discuss in detail the correspondence in linearized gravity between this CT decomposition of the KST system and the BSSN equations. In Section III we show that by introducing extra variables to make the BSSN system first order in time and space one can get strongly and symmetric hyperbolic systems provided that the lapse is appropriately densitized. We end with a discussion of the relevance of these results for numerical simulations. 


\section{THE BSSN-KST CORRESPONDENCE}

In this section, we show that for the case of linearization around flat space-time in Cartesian coordinates the BSSN equations are equivalent (in a sense to be defined below) to the linearized KST equations with an appropriate choice of parameters. As a byproduct of our construction, we present a CT decomposition of the KST system that shares its same level of hyperbolicity, even at the nonlinear level.

\section{A. The KST family of equations}

Starting from the ADM equations, KST derive a family of strongly and symmetric hyperbolic first-order evolution equations for the three-metric $\left(g_{i j}\right)$, the extrinsic curvature $\left(K_{i j}\right)$, and the spatial derivatives of the three-metric $\left(d_{k i j} \equiv \partial_{k} g_{i j}\right)$, which generalizes previous well posed formulations 11, 12]. A priori prescribing the densitized lapse $\exp (Q)$ as a function of spacetime, the lapse $N$ is given by

$$
N=g^{\sigma} e^{Q}
$$

where $g$ is the determinant of the three-metric and $\sigma$ a parameter. The shift vector, $\beta^{i}$, is also assumed to be prescribed as a function of spacetime. Here we use the same notation as in KST except that we define the derivative operator along the normal to the hypersurfaces as

$$
\partial_{0}=\frac{1}{N}\left(\partial_{t}-£_{\beta}\right)
$$

The vacuum evolution equations have the form

$$
\begin{aligned}
\partial_{0} g_{i j} & =-2 K_{i j}, \\
\partial_{0} K_{i j} & =-\frac{1}{2} \partial^{k} d_{k i j}+\frac{1}{2} \partial^{k} d_{(i j) k}+\frac{1}{2} g^{a b} \partial_{(i} d_{|a b| j)}-\left(\frac{1}{2}+\sigma\right) g^{a b} \partial_{(i} d_{j) a b} \\
& +\zeta g^{a b} C_{a(i j) b}+\gamma g_{i j} C-e^{-Q} \partial_{i} \partial_{j}\left(e^{Q}\right)+\mathcal{R}_{i j}, \\
\partial_{0} d_{k i j} & =-2 \partial_{k} K_{i j}+\eta g_{k(i} C_{j)}+\chi g_{i j} C_{k}+\mathcal{R}_{k i j},
\end{aligned}
$$

where the constraint variables

$$
\begin{array}{cl}
C=\frac{1}{2} g^{a b} \partial^{k}\left(d_{a b k}-d_{k a b}\right)+\mathcal{R}, & \text { (Hamiltonian constraint) } \\
C_{j}=\partial^{a} K_{a j}-g^{a b} \partial_{j} K_{a b}+\mathcal{R}_{j}, & \text { (momentum constraint) } \\
C_{k i j}=d_{k i j}-\partial_{k} g_{i j}, & \text { (definition of } \left.d_{k i j}\right) \\
C_{l k i j}=\partial_{[l} d_{k] i j} . & \text { "closedness" of } \left.d_{k i j}\right)
\end{array}
$$

have been added to the right-hand side of the evolution equations with some free parameters $\zeta, \gamma, \eta, \chi$. Here, $\partial^{k} \equiv g^{k l} \partial_{l}$ and $b_{j}, d_{k}$ are defined as the traces of $d_{k i j}$ :

$$
b_{j}=g^{k i} d_{k i j}, \quad d_{k}=g^{i j} d_{k i j} .
$$

The "remainder" terms $\mathcal{R}, \mathcal{R}_{i}, \mathcal{R}_{i j}$ and $\mathcal{R}_{k i j}$ are homogeneous polynomials of degree 2 in $d_{k i j}, \partial_{i} Q$ and $K_{i j}$, where the coefficients may depend on $g_{i j}$. Finally, the Lie derivative of the symbols $d_{k i j}$ is

$$
£_{\beta} d_{k i j}=\beta^{l} \partial_{l} d_{k i j}+d_{l i j} \partial_{k} \beta^{l}+2 d_{k l(i} \partial_{j)} \beta^{l}+2 g_{l(i,} \partial_{j)} \partial_{k} \beta^{l} .
$$

The evolution system has characteristic speeds $\left\{0, \pm 1, \pm \sqrt{\lambda_{1}}, \pm \sqrt{\lambda_{2}}, \pm \sqrt{\lambda_{3}}\right\}$, where

$$
\begin{aligned}
& \lambda_{1}=2 \sigma \\
& \lambda_{2}=1+\chi-\frac{1}{2}(1+\zeta) \eta+\gamma(2-\eta+2 \chi), \\
& \lambda_{3}=\frac{1}{2} \chi+\frac{3}{8}(1-\zeta) \eta-\frac{1}{4}(1+2 \sigma)(\eta+3 \chi) .
\end{aligned}
$$


It should be noted that these are the characteristic speeds with respect to the $\partial_{0}$ operator. This means that $\lambda=0,1$ correspond to propagation along the normal to the hypersurfaces or the light cone, respectively (physical speeds). The characteristic speeds with respect to the $\partial_{t}$ operator are obtained from these after the transformation

$$
\mu \mapsto N \mu+\beta^{i} n_{i}
$$

where $n^{i}$ is the direction of the corresponding characteristic mode.

The conditions under which the system is completely ill posed (CIP), WH or strongly hyperbolic (SH) were found by KST. The system is CIP if any of the above speeds is complex, while it is WH if $\lambda_{j} \geq 0$ for $j=1,2,3$ but one of the conditions (5, 6) below is violated. For example, if the parameters $(\zeta, \gamma, \eta, \chi)$ are zero the dynamics is equivalent to the ADM equations written in first order form with fixed densitized lapse and fixed shift (which are WH). If $\sigma=0$ as well, then the system is equivalent to the ADM equations with fixed lapse and shift (which are also WH).

Finally, the system is $\mathrm{SH}$ provided that

$$
\begin{aligned}
& \lambda_{j}>0, \quad \text { for } j=1,2,3, \\
& \lambda_{3}=\frac{1}{4}\left(3 \lambda_{1}+1\right) \text { if } \lambda_{1}=\lambda_{2} .
\end{aligned}
$$

Here by $\mathrm{SH}$ it is meant that the principal part has a complete set of eigenvectors with real eigenvalues. Provided there is a smooth and uniformly bounded symmetrizer, this ensures well posedness [2].

In KST seven extra parameters are introduced and used to make changes of variables in $K_{i j}$ and $d_{k i j}$. When performing this change of variables the constraint $C_{k i j}=0$ is also used in order to trade spatial derivatives of the three-metric for $d_{k i j}$. Thus, the equations with the new variables have different solutions off the constraint surface. However, one can see that at the linear level this change of variables does not involve the addition of constraints.

\section{B. Conformal trace-less decomposition of the KST system}

We start by performing a CT of the KST variables:

$$
\begin{aligned}
g_{i j} & =e^{4 \phi} \tilde{g}_{i j} \\
K_{i j} & =e^{4 \phi}\left(A_{i j}+\frac{1}{3} \tilde{g}_{i j} K\right), \\
d_{k i j} & =e^{4 \phi}\left(2 e_{k i j}+\frac{3}{5} \tilde{g}_{k(i)} \Gamma_{j)}-\frac{1}{5} \tilde{g}_{i j} \Gamma_{k}+\frac{1}{3} \tilde{g}_{i j} d_{k}\right) .
\end{aligned}
$$

Here $\tilde{g}_{i j}$ has unit determinant, $A_{i j}$ is trace-less and $e_{k i j}$ is trace-less over all pairs of indices, and $\Gamma_{j}$ is defined by $\Gamma_{j}=b_{j}-d_{j} / 3$. In terms of the Christoffel symbols $\tilde{\Gamma}_{i j}^{k}$ belonging to the conformal metric $\tilde{g}_{i j}, \Gamma_{j}$ can also be expressed as $\left(\tilde{\partial}^{i} \equiv \tilde{g}^{i k} \partial_{k}\right)$

$$
\Gamma_{j}=\tilde{g}^{k i} \tilde{\Gamma}_{j k i}=\tilde{\partial}^{i} \tilde{g}_{i j},
$$

which is the additional variable used in the BSSN system (actually, in the original version by Shibata and Nakamura, the variable $F_{j}=\delta^{k i} \partial_{k} \tilde{g}_{i j}$ is used instead of $\Gamma_{j}$, in the version by Baumgarte and Shapiro, $\Gamma^{k}=\tilde{g}^{k j} \Gamma_{j}$ is used.)

With respect to this decomposition, the KST system (2-4) becomes, up to lower order terms ("l.o.") quadratic in $d_{k i j}, \partial_{i} Q$ and $K_{i j}$

$$
\begin{aligned}
\partial_{0} \phi & =-\frac{1}{6} K \\
\partial_{0} \tilde{g}_{i j} & =-2 A_{i j} \\
\partial_{0} K & =\left(1+\frac{3}{2} \gamma\right) \partial^{k} \Gamma_{k}-\left(\frac{2}{3}+\gamma+\sigma\right) \partial^{k} d_{k}-e^{-Q} \partial^{k} \partial_{k}\left(e^{Q}\right)+l . o ., \\
\partial_{0} A_{i j} & =-\partial^{k} e_{k i j}+(1+\zeta) \partial^{k} e_{(i j) k}+e^{-4 \phi}\left[\frac{1}{20}(5-9 \zeta) \partial_{(i} \Gamma_{j)}-\left(\frac{1}{6}+\sigma\right) \partial_{(i} d_{j)}-e^{-Q} \partial_{i} \partial_{j}\left(e^{Q}\right)\right]^{T F}+(l . o .)_{i j},(1) \\
\partial_{0} d_{k} & =(\eta+3 \chi) \tilde{\partial}^{s} A_{s k}-\frac{2}{3}(\eta+3 \chi+3) \partial_{k} K+(l . o .)_{k}, \\
\partial_{0} \Gamma_{j} & =\left(\frac{5}{3} \eta-2\right) \tilde{\partial}^{s} A_{s j}-\frac{10}{9} \eta \partial_{j} K+(l . o .)_{j}, \\
\partial_{0} e_{k i j} & ==-\partial_{k} A_{i j}+\frac{3}{5} \tilde{g}_{k(i} \tilde{\partial}^{s} A_{j) s}-\frac{1}{5} \tilde{g}_{i j} \tilde{\partial}^{s} A_{s k}+(l . o .)_{k i j},
\end{aligned}
$$


where the superscript $T F$ denotes the trace-free part. Here, for simplicity, we have also assumed that the shift vanishes. However, a generalization in the presence of shift can also be worked out.

Notice that in the derivation of Eqs. (7 13 ) we have used the constraints $C_{k i j}=0$ in order to re-express first order spatial derivatives of $g_{i j}$ in terms of $d_{k i j}$ in a way that ensures that the principal part of the transformed equations has the same spectrum as the original KST system. Therefore, for a given choice of parameters both systems have the same level of hyperbolicity. However, in general the dynamics of the evolution Eqs. (77 13) is not identical to the dynamics of the KST Eqs. (2-4) if we are off the constraint surface $C_{k i j}=0$. A similar situation occurs in KST when the variables $K_{i j}$ and $d_{k i j}$ are redefined through a seven-parameter family of change of variables.

\section{Correspondence}

We start by looking at the linearized equations around flat spacetime, written in Cartesian coordinates. One can see

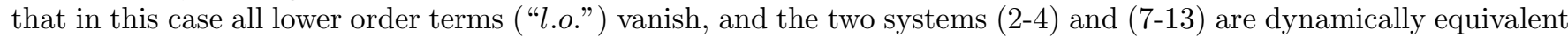
(i.e. have the same set of solutions, even off the constraint surface). In the following, we will call the linearized version of equations (713) system A.

Since the BSSN system has second order spatial derivatives and the KST system has only first order spatial derivatives, care needs to be exercised concerning the constraints that materialize the correspondence of the spatial derivatives. Basically, we need to show that if we start with initial data that is on the submanifold of constraints that relate the second spatial derivatives to first spatial derivatives of extra variables (all solutions of the BSSN system automatically lie on this submanifold), we automatically remain on it. Otherwise, no correspondence will be possible.

Consider the constraints

$$
\tilde{C}_{k i j}=e_{k i j}-\frac{1}{2} \partial_{k} \tilde{g}_{i j}+\frac{3}{10} \delta_{k(i} \partial^{s} \tilde{g}_{j) s}-\frac{1}{10} \delta_{i j} \partial^{s} \tilde{g}_{k s}
$$

Using the linearized version of evolution equations (8) and (13), one can see that these constraints are constant in time,

$$
\partial_{0} \tilde{C}_{k i j}=0
$$

independently of whether or not the remaining constraints are satisfied. The constraints $\tilde{C}_{k}=d_{k}-12 \partial_{k} \phi$ are constant in time as well, provided that $\eta+3 \chi=0$. Therefore, if we give initial data with $\tilde{C}_{k i j}=0$ and $\tilde{C}_{k}=0$, the equations

$$
\begin{aligned}
e_{k i j} & =\frac{1}{2} \partial_{k} \tilde{g}_{i j}-\frac{3}{10} \delta_{k(i} \partial^{s} \tilde{g}_{j) s}+\frac{1}{10} \delta_{i j} \partial^{s} \tilde{g}_{k s} \\
d_{k} & =12 \partial_{k} \phi
\end{aligned}
$$

hold at all times, provided that $\eta+3 \chi=0$. In this case, we can replace $e_{k i j}$ and $d_{k}$ by the right-hand side of equations (14) and (15) everywhere they appear in the linearized version of equations (7) 12 and forget about the evolution equations for the 15 variables $e_{k i j}, d_{k}$. The evolution equations for $K$ and $A_{i j}$ become

$$
\begin{aligned}
\partial_{0} K & =\left(1+\frac{3}{2} \gamma\right) \partial^{k} \Gamma_{k}-8\left(1+\frac{3}{2} \gamma+\frac{3}{2} \sigma\right) \partial^{k} \partial_{k} \phi-e^{-Q} \partial^{k} \partial_{k}\left(e^{Q}\right) \\
\partial_{0} A_{i j} & =-\frac{1}{2} \partial^{k} \partial_{k} \tilde{g}_{i j}+\left[\partial_{(i} \Gamma_{j)}+\frac{1}{20}(15+9 \zeta)\left(\partial_{(i} \partial^{s} \tilde{g}_{j) s}-\partial_{(i} \Gamma_{j)}\right)-2(1+6 \sigma) \partial_{i} \partial_{j} \phi-e^{-Q} \partial_{i} \partial_{j}\left(e^{Q}\right)\right]^{T F} \cdot
\end{aligned}
$$

In the following, we will call the system which constitutes the linearized version of equations (77,8, 12) and the equations $(16,17)$ system B. If initial data is chosen for system B and one takes the same initial data for system A with $d_{k}$ and $e_{k i j}$ given by equations (14.15), the two systems will yield the same result upon evolution (if there are no boundaries or if we have time-like boundaries to which $\partial_{0}$ is tangential.)

If we compare system B with the generalization of the BSSN system by Alcubierre et al. [9] in the linearized regime, we see that the two agree if the KST parameters are

$$
\zeta=-\frac{5}{3}, \quad \gamma=-\frac{2}{3}, \quad \eta=\frac{6}{5} m, \quad \chi=-\frac{2}{5} m .
$$

Notice that since [9] reduces to BSSN [7], 8] when $m=1$, system B agrees with the original BSSN as well for these values of the parameters. Notice also that for the equivalence to be true one has to use in BSSN the same gauge conditions used in $\mathrm{KST}$ (Eq. (1) with arbitrary $\sigma$ ). 
For example, the choice $\gamma=-\frac{2}{3}$ eliminates the Ricci scalar appearing in the evolution equation for $K$, and choosing $\eta=\frac{6}{5}$ eliminates the term $\partial^{s} A_{s k}$ in the evolution equation for the variable $\Gamma_{j}$, as in the traditional BSSN system (while in [9] the parameter $m$ is allowed to vary). Therefore, the linearized BSSN equations are dynamically equivalent to the linearized KST system with the choice of parameters given by (18), provided that the constraints (14, 15) are satisfied. In particular, any solution to the linearized BSSN equations (even one that does not satisfy the Hamiltonian or momentum constraint, or the constraint that corresponds to the definition of $\Gamma_{j}$ ) gives a solution to the linearized KST system with (18) if we define $d_{k}$ and $e_{k i j}$ according to (14,15).

Now an interesting question is whether or not the KST subfamily that corresponds to the linearized BSSN system is SH. Recalling Section II, it turns out that the characteristic speeds are plus or minus the square root of

$$
\lambda_{1}=2 \sigma, \quad \lambda_{2}=\frac{1}{3}(4 m-1), \quad \lambda_{3}=m, \quad \lambda_{4}=1 .
$$

In particular, we see that densitizing the lapse with $\sigma>0$ in (18) corresponds to a SH system as long as $m>1 / 4$. In particular, if $\sigma=1 / 2$ and $m=1$, the system has only physical speeds $(\lambda=0,1)$. It is also worthwhile pointing out that in this (linear, constant coefficient) case well posedness can be shown for the SH families, since all is needed is the boundedness of the symmetrizer, which can be shown to hold.

One might wonder whether the parameters (18) (with $\sigma=1 / 2$ ) correspond to the ones found empirically by KST to have better stability properties. The answer is no, since the one-parameter subfamily (18) has no intersection with the two-parameter Einstein-Christoffel (EC) [12] generalization used in the numerical simulations of KST.

\section{HYPERBOLIC FIRST ORDER ENLARGEMENTS OF THE BSSN SYSTEM}

We were not able to find a way in the non-linear case to get rid of the extra variables $e_{k i j}$ and $d_{k}$ when lower order terms are present in the equations. We will therefore not prove a correspondence between the KST and BSSN systems but, instead, a correspondence between the BSSN system and certain SH or symmetric hyperbolic formulations closely related to one by Friedrich and Rendall 10. Starting with the (non-linear) BSSN system with vanishing shift (see below for the case of non-vanishing shift) we are able to show the following result: by introducing 18 extra variables, the BSSN system can be written as a system which is first order in time and space and whose principal part looks very similar to the one of the CT decomposition of the KST system. Drawing from our experience with the latter, one can then show that the new first order system has the same characteristic speeds as system B with the choice of parameters (18). With a specific choice of parameters $\sigma$ and $m$ this system has the same level of hyperbolicity as the KST one.

The non-linear BSSN equations with zero shift have the form

$$
\begin{aligned}
\partial_{0} \phi & =-\frac{1}{6} K \\
\partial_{0} \tilde{g}_{i j} & =-2 A_{i j} \\
\partial_{0} K & =-12 \sigma \partial^{k} \partial_{k} \phi-e^{-Q} \partial^{k} \partial_{k}\left(e^{Q}\right)+\text { l.o. } \\
\partial_{0} A_{i j} & =e^{-4 \phi}\left[-\frac{1}{2} \tilde{\partial}^{k} \partial_{k} \tilde{g}_{i j}+\partial_{(i} \Gamma_{j}-2(1+6 \sigma) \partial_{i} \partial_{j} \phi-e^{-Q} \partial_{i} \partial_{j}\left(e^{Q}\right)\right]^{T F}+(\text { l.o. })_{i j}, \\
\partial_{0} \Gamma_{i} & =2(m-1) \tilde{\partial}^{s} A_{s i}-\frac{4 m}{3} \partial_{i} K+(\text { l.o. })_{i},
\end{aligned}
$$

where $\partial_{0}=\partial_{t} / N$, and now "l.o." denote terms that contain only derivatives of $\phi, \tilde{g}_{i j}, Q$ up to first order, and no derivatives of the other variables. Here, the lapse $N$ and the densitized lapse $\exp (Q)$ are related to each other as in equation (11). In terms of the BSSN variables,

$$
N=e^{12 \sigma \phi+Q} .
$$

For generality, we have also added the momentum constraint to the evolution equation for $\Gamma_{i}$ with an arbitrary factor $m$ as in Alcubierre et. al. [9].

Our aim is to recast this system of equations into a first order system by introducing extra variables,

$$
d_{k}=12 \partial_{k} \phi, \quad \tilde{d}_{k i j}=\partial_{k} \tilde{g}_{i j},
$$

and using only the constraints that are associated to the definition of these extra variables. Using the fact that

$$
\left[\partial_{0}, \partial_{k}\right]=\frac{N_{k}}{N} \partial_{0}=\left(12 \sigma \partial_{k} \phi+\partial_{k} Q\right) \partial_{0}=\left(\sigma d_{k}+\partial_{k} Q\right) \partial_{0},
$$


and Eqs. (19,20), we obtain the following evolution equations for $d_{k}$ and $\tilde{d}_{k i j}$ :

$$
\begin{aligned}
\partial_{0} d_{k} & =-2\left(\partial_{k}+\sigma d_{k}+\partial_{k} Q\right) K, \\
\partial_{0} \tilde{d}_{k i j} & =-2\left(\partial_{k}+\sigma d_{k}+\partial_{k} Q\right) A_{i j} .
\end{aligned}
$$

The constraints associated to the definition of $d_{k}$ and $\tilde{d}_{k i j}, \tilde{C}_{k}$ and $\tilde{C}_{k i j}$, satisfy

$$
\begin{aligned}
\partial_{0} \tilde{C}_{k} & =-2 \sigma K \tilde{C}_{k}, \\
\partial_{0} \tilde{C}_{k i j} & =-2 \sigma A_{i j} \tilde{C}_{k},
\end{aligned}
$$

independently on whether or not the remaining constraints are satisfied. Since the evolution equations for $\tilde{C}_{k}$ and $\tilde{C}_{k i j}$ form a closed system, the equations that define $d_{k}$ and $\tilde{d}_{k i j}$ are satisfied automatically during evolution (provided that they are satisfied initially and appropriate conditions are given at time-like boundaries.)

Thus, we can enlarge system (19,23) by adding the evolution equations (26,27) above and by replacing $12 \partial_{k} \phi$ by $d_{k}$ and $\partial_{k} \tilde{g}_{i j}$ by $\tilde{d}_{k i j}$, respectively. The resulting system is

$$
\begin{aligned}
\partial_{0} \phi & =-\frac{1}{6} K \\
\partial_{0} \tilde{g}_{i j} & =-2 A_{i j}, \\
\partial_{0} K & =-\sigma \partial^{k} d_{k}-e^{-Q} \partial^{k} \partial_{k}\left(e^{Q}\right)+\text { l.o. }, \\
\partial_{0} A_{i j} & =-\frac{1}{2} \partial^{k} \tilde{d}_{k i j}+e^{-4 \phi}\left[\partial_{(i} \Gamma_{j}-\left(\frac{1}{6}+\sigma\right) \partial_{(i} d_{j)}-e^{-Q} \partial_{i} \partial_{j}\left(e^{Q}\right)\right]^{T F}+(l . o .)_{i j}, \\
\partial_{0} \Gamma_{j} & =2(m-1) \tilde{\partial}^{s} A_{s j}-\frac{4 m}{3} \partial_{j} K+(\text { l.o. })_{j}, \\
\partial_{0} d_{k} & =-2 \partial_{k} K+(\text { l.o. })_{k}, \\
\partial_{0} \tilde{d}_{k i j} & =-2 \partial_{k} A_{i j}+(\text { l.o. })_{k i j},
\end{aligned}
$$

where after the replacements mentioned above, no derivatives of the dynamical variables appear in "l.o.". Analyzing the level of hyperbolicity, it turns out that the system (30-36) has the same characteristic speeds as the KST system with the choice (18) and the same level of hyperbolicity. In particular, it is SH if $\sigma>0$ and $m>1 / 4$, and has only physical characteristic speeds if $\sigma=1 / 2$ and $m=1$.

Furthermore, it is easy to see that if the relation

$$
2 \sigma=\frac{1}{3}(4 m-1)
$$

holds and $m>1$, the principal part of the system is symmetric with respect to the inner product associated with

$$
(u, u) \equiv \phi^{2}+\tilde{g}^{i j} \tilde{g}_{i j}+\frac{3}{4 m-1} K^{2}+A^{i j} A_{i j}+\frac{e^{-4 \phi}}{2(m-1)}\left(\Gamma^{k} \Gamma_{k}-\frac{4 m}{3} \Gamma^{k} d_{k}+\frac{4 m^{2}}{9} d^{k} d_{k}\right)+\frac{e^{-4 \phi}}{4}\left(d^{k} d_{k}+\tilde{d}^{k i j} \tilde{d}_{k i j}\right),
$$

where $u=\left(\phi, \tilde{g}_{i j}, K, A_{i j}, d_{k}, \Gamma_{j}, \tilde{d}_{k i j}\right)^{T}$ and where indices are raised with the metric $\tilde{g}_{i j}$. A point to be remarked is that this symmetric hyperbolic subfamily has superluminal speeds, since we have to impose the condition $m>1$ and Eq. (37) must hold. In particular, we cannot recover the original BSSN system $(m=1)$ but, instead, its generalization by Alcubierre et. al..

To summarize, we have shown that any version of BSSN which has the form (19-23) can be rewritten as a first order system in time and space by introducing the extra variables (25). Provided that initially the constraints associated to the definition of the new variables are satisfied, this first order system is equivalent to the BSSN system chosen. For a fixed densitized lapse, the system is SH if $\sigma>0$ and $m>1 / 4$. If $m>1$ and we choose to densitize the lapse according to Eq. (37), the system is even symmetric hyperbolic. At this point it is worthwhile pointing out that a similar enlargement to a SH first order system does not work in the ADM case, even after densitizing the lapse. In order to get a SH system, one must add the Hamiltonian or momentum constraints to the evolution equations.

If the shift is non-trivial, one can still obtain the above enlargement. But in this case, divergence terms of the conformal metric $\tilde{g}_{i j}$ have to be substituted by $\Gamma_{i}$ 's in such a way that the equations have the form (19-23) with $\partial_{0}=\left(\partial_{t}-\beta^{k} \partial_{k}\right) / N$ and where the lower order terms ("l.o.") may depend on $\beta^{i}$. In this case, the enlargement is SH or symmetric hyperbolic under the same conditions as is the zero shift case provided the shift is a prescribed function.

Frittelli and Reula (FR) have also presented well posed CT formulations of Einstein's equations [13] that have attractive properties (for example, only physical characteristic speeds), but they do not seem to reduce to the BSSN system in the sense described above due to the way the constraints are added in their construction. 


\section{SUMMARY}

We have shown that at the linearized level the BSSN system with fixed densitized lapse and fixed shift is equivalent to a SH subfamily of the KST formulation, while at the nonlinear level it is equivalent to certain $\mathrm{SH}$ or symmetric hyperbolic system. This equivalence is analogous to the one between the wave equation written as first order in time and space, or first order in time but second order in space.

The hyperbolicity of BSSN (and any other system) depends delicately on how the equations are written. Small substitutions of some equations in others are enough to spoil hyperbolicity. This may account for several anecdotal reports that rewriting part of the BSSN equations appears to improve or worsen the numerical behavior. Having established that this system is hyperbolic opens the possibility of rigorously showing that the resulting numerical schemes constructed upon discretization are stable in the sense of Lax's theorem, and therefore the resulting codes are convergent. This property is not available at present for $\mathrm{WH}$ formulations, including the ADM equations or even small modifications of the BSSN system considered here. Another advantage is that one could also derive consistent boundary conditions for the BSSN equations or show well posedness by looking at the first order hyperbolic enlargement and later reducing the result back to second order in space. This therefore lays mathematical grounds on which to understand and make use of the advantages in numerical implementations of the BSSN equations.

\section{ACKNOWLEDGMENTS}

This work was supported in part by grant nsf-phy9800973, by the Horace C. Hearne Jr. Institute of Theoretical Physics, the Swiss National Science Foundation, and Fundación Antorchas.

[1] O. A. Reula, Living Rev. Rel. 1, 3 (1998).

[2] B. Gustafsson, H.O. Kreiss, J. Oliger, Time dependent problems and difference methods (Wiley, New York, 1995).

[3] H. Friedrich and G. Nagy, Commun. Math. Phys. 204, 691 (1999).

[4] B Szilagyi and J. Winicour, gr-qc/0205044; G. Calabrese, L. Lehner and M. Tiglio, Phys. Rev. D 65, 104031 (2002); J. M. Bardeen and L. T. Buchman, Phys. Rev. D 65, 064037 (2002); B. Szilagyi, B. Schmidt, and J. Winicour, Phys. Rev. D 65, 064015 (2002); M. Iriondo and O. Reula, ibid. 65, 044024 (2002)); J.M. Stewart, Class. Quantum Grav. 15, 2865 (1998).

[5] L.E. Kidder, M.A. Scheel, and S.A. Teukolsky, Phys. Rev. D 64, 064017 (2001).

[6] G. Calabrese, J. Pullin, O. Sarbach, and M. Tiglio, Convergence and stability in numerical relativity, in preparation.

[7] M. Shibata and T. Nakamura, Phys. Rev. D 52, 5428 (1995).

[8] T.W. Baumgarte and S.L. Shapiro, Phys. Rev. D 59, 024007 (1998)

[9] M. Alcubierre et al., Phys. Rev. D 62, 124011 (2000).

[10] H. Friedrich and A. Rendall, in Einstein's Field Equations and their Physical Interpretation, edited by B. G. Schmidt (Springer, Berlin, 2000), gr-qc/000207.

[11] S. D. Hern, Ph.D. thesis, University of Cambridge, 1999, gr-qc 0004036; S. Frittelli and O. A. Reula, Phys. Rev. Lett. 76, 4667 (1996).

[12] A. Anderson and J.W. York, Jr., Phys. Rev. Lett. 82, 4384 (1999).

[13] S. Frittelli and O. A. Reula, J. Math. Phys. 40, 5143 (1999). 